\title{
$\Delta S=1$ Weak Transitions in Skyrme model Generalized up to Quartic Terms
}

\author{
Rajesh Kumar \\ Department of Physics, Govt. College, Faridabad, Haryana, India \\ Corresponding Author : dr.rkumar65@gmail.com
}

\begin{abstract}
We investigate $\Delta \mathrm{S}=1$ weak transition in the light of most general Skyrme type lagrangian containing terms up to quartic terms in field gradients. The additional parameters in the lagrangian are taken from pion-pion scattering data. Our decay amplitudes for anticommutator term in the Skyrme lagrangian shows much improvement over the Skyrme values. S-wave decay amplitudes are also reasonably reproduced in the model.
\end{abstract}

Keywords : Chiral Symmetry, Skyrmion, Heavier Mesons

\section{INTRODUCTION}

In Skyrme model, baryons emerge as solitonic solutions of non-linear pion field ${ }^{1}$. ANW ${ }^{2}$ calculated the physical properties of nucleons in the model within 30\% accuracy. Since then, there has been motivation for studying mass spectrum and baryon static properties by including

1) mesons heavier than pion $^{3-10}$

2) terms higher order in field derivatives ${ }^{11-14}$

in the lagrangian. The extension of the model to strange sector to account for the large strange quark mass has been done by including chiral symmetry breaking terms whose complete survey is given in ref. ${ }^{15}$. If the Skyrme model has to emerge as an effective theory in the low limit, it must predict weak decays because as quoted by Scoccola ${ }^{16}$ these decays have been the least understood aspect of low energy weak interactions.

$\mathrm{PT}^{17}$ calculated the weak decay amplitudes of hyperons in Skyrme model by constructing a weak Hamiltonian in form of current-current interaction and using the baryon wave functions of the model. In these calculations $\Delta \mathrm{I}=1 / 2$ rule (octet dominance) is well satisfied. These decay amplitudes were compared with quark model (QM) values e.g. for transition

$$
\Sigma^{+} \rightarrow \mathrm{P}+\pi^{0}
$$

Skyrme model decay amplitude for a chirally symmetric lagrangian is

$$
\mathrm{A}=0.65 \times 10^{-3} \mathrm{GeV}^{3}
$$

While for broken chiral symmetry, this amplitude is

$$
\mathrm{A}=0.3 \times 10^{-3} \mathrm{GeV}^{3}
$$

The $\mathrm{QM}^{18}$ value for same transition is

$$
\mathrm{A}=3.15 \times 10^{-3} \mathrm{GeV}^{3}
$$

It can be seen that chirally symmetric case(Eq.1) value is about five times smaller than QM value(Eq.3) and broken chiral symmetry(Eq.2) is about ten times smaller. It seems that

the origin of failure in reproducing the absolute weak decay amplitudes lies in the small values $F_{\pi}$ (pion decay constant) which was used to predict the hyperon spectra ${ }^{19-20}$. A similar study was carried out by Kondo et.al. ${ }^{21}$ with bound state approach to strangeness in the model. The results obtained were qualitatively similar to those obtained by $\mathrm{PT}^{17}$ but quantitatively different. $\mathrm{PT}^{17}$ quoted that lagrangian chosen

by them is the least part of the physical one. Therefore, in this paper, the aim is to investigate the non-leptonic weak decay amplitudes of hyperons in Skyrme model 
by considering a generalized lagrangian up to quartic terms in field gradients.

Such a most general Skyrme type lagrangian was proposed

by Weinberg22. One term of this lagrangian i.e. $\left[\operatorname{Tr}\left(\partial^{\mu} U \partial_{\mu} U^{\dagger}\right)\right]^{2}$

was considered by Lacombe et.el. ${ }^{23}$ in the Skyrme model

for studying $\mathrm{NN}$ interaction and found that this term produces significant attraction in the intermediate range. It is, imperative, now to investigate the effects of additional terms proposed by Weinberg in the lagrangian on weak non-leptonic hyperon decay amplitudes. The paper is organized as follows

- In section 2, SU(3) Skyrme model is discussed by considering the most general Skyrme type lagrangian. For calculating the various integrals, arctan ansatz is used for chiral angle.

- $\quad$ Noether currents are discussed in section 3

- In section 4 , current $\times$ current weak Hamiltonian is derived in terms of SU(3) Dfunctions.

- $\quad$ Section 5 concludes the paper.

\section{SU(3) Skyrme Model}

The Skyrme lagrangian density ${ }^{7}$ is

$$
\begin{aligned}
& \mathcal{L}_{\mathrm{sk}}=\frac{f_{\pi}^{2}}{4} \operatorname{Tr}\left(\partial_{\mu} U^{\dagger} \partial^{\mu} U\right)+\frac{1}{32 e^{2}} \operatorname{Tr}\left[\partial_{\mu} U U^{\dagger}, \partial_{\nu} U U^{\dagger}\right]^{2} \\
& f_{\pi}=\frac{F_{\pi}}{2} \text { is pion decay constant. }
\end{aligned}
$$

Weinberg proposed that most general Skyrme type lagrangian density up to quartic in field derivatives is given by

$$
\mathcal{L}_{\mathrm{w}}=\mathcal{L}_{\mathrm{sk}}+\frac{\beta}{32 e^{2}} \operatorname{Tr}\left[\partial_{\mu} U U^{\dagger}, \partial_{\nu} U U^{\dagger}\right]_{+}^{2}+\frac{\gamma}{8 e^{2}}\left[\operatorname{Tr}\left(\partial_{\mu} U^{\dagger} \partial^{\mu} U\right)\right]^{2}
$$

$\beta$ and $\Upsilon$ are taken from $\pi-\pi$ scattering data. Effective lagrangian is chosen as

$$
\begin{aligned}
& \mathrm{L}=\int \mathcal{L} \mathrm{d}^{3} \mathrm{r}+\mathrm{N}_{\mathrm{c}} \Gamma \\
& \\
& \mathcal{L}=\mathcal{L}_{\mathrm{w}}+\frac{f_{\pi}^{2}}{8}\left(m_{\pi}^{2}+m_{\eta}^{2}\right) \operatorname{Tr}\left(\mathrm{U}+\mathrm{U}^{\dagger}-2\right)+\frac{\sqrt{3}}{6} \mathrm{f}_{\pi}^{2}\left(\mathrm{~m}_{\pi}^{2}-\mathrm{m}_{\mathrm{k}}^{2}\right) \operatorname{Tr} \lambda_{8}\left(\mathrm{U}+U^{\dagger}\right) \\
& \Gamma=-\frac{i}{240 \pi} 2 \int d \Sigma^{\mu \nu \rho \sigma \tau} \operatorname{Tr}\left(\partial \mu U U^{\dagger} \partial \nu U U^{\dagger} \partial \rho U U^{\dagger} \partial \sigma U U^{\dagger} \partial \tau U U^{\dagger}\right)
\end{aligned}
$$

The lagrangian (Eq.6) has the static solution

$$
\mathrm{U}_{0}(\mathrm{r})=\exp \left(\lambda_{j} \frac{r_{j}}{r} F(r)\right)
$$

The model is quantized by making the unitary transformation

$$
\mathrm{U}(\mathrm{r}, \mathrm{t})=\mathrm{A}(\mathrm{t}) \mathrm{U}_{0}(\mathrm{r}) \dot{A}^{-1}(\mathrm{t})
$$

where $\mathrm{A}(\mathrm{t})$ is time dependent $\mathrm{SU}(3)$ matrix which defines general velocities $a$

$$
\mathrm{A}^{-1} \dot{A}=\frac{1}{2} i \sum_{a=1}^{8} \lambda_{a} \dot{a}_{a}
$$

$\lambda a$ are the Gell- Mann SU (3) matrices. The chiral angle $\mathrm{F}(\mathrm{r})$ is subjected to boundary conditionsThe lagrangian (Eq.5) is, now, transformed to

$$
\mathrm{L}=-\mathrm{M}+\frac{1}{2} \mathrm{I}_{1} \sum_{i=1}^{3} \dot{a}^{2}+\frac{1}{2} \mathrm{I}_{2} \sum_{j=4}^{7} \dot{a}^{2}+\frac{N}{2} \frac{B}{\sqrt{3}} \dot{a}_{8}+\frac{1}{2} \Upsilon_{\mathrm{m}} D_{88}
$$

where $\mathrm{M}$ is the classical soliton mass. The quantities $\mathrm{I}_{1}$ and $\mathrm{I}_{2}$ represent the moment of inertia for rotations in co-ordinate space and flavor rotations in the directions of strangeness respectively with

$$
\begin{aligned}
& \mathrm{M}=2 \pi \frac{f_{\pi}}{e} \int x^{2} d x\left[\left(F^{\prime 2}+\frac{\sin ^{2} F}{x^{2}}\right)+\frac{\sin ^{2} F}{x^{2}}\left(2 F^{\prime 2}+\frac{\sin ^{2} F}{x^{2}}\right)\right. \\
& \left.-\frac{\beta}{2}\left(F^{\prime 4}+2 \frac{\sin ^{4} F}{x^{2}}\right)-\gamma\left(F^{\prime 2}+2 \frac{\sin ^{2} F}{x^{2}}\right)+\mu^{2}(1-\cos F)\right] \\
& \mathrm{I}_{1}=\frac{8 \pi}{3} \frac{1}{e^{3} f_{\pi}} \int x^{2} \sin ^{2} F\left[1+\left(F^{\prime 2}+\frac{\sin ^{2} F}{x^{2}}\right)-\beta \frac{\sin ^{2} F}{x^{2}}\right. \\
& \left.-2 \Upsilon\left(F^{\prime 2}+2 \frac{\sin ^{2} F}{x^{2}}\right)\right] d x \\
& \mathrm{I}_{2}=\frac{\pi}{2 e^{3} f_{\pi}} \int x^{2}(1-\cos F)\left[4+\{1-(\beta+8 \gamma)\}\left(F^{\prime 2}+2 \frac{\sin ^{2} F}{x^{2}}\right)\right] d x \\
& \Upsilon_{m}=\frac{16 \pi}{3}\left(m_{k}^{2}-m_{\pi}^{2}\right) \frac{1}{e^{3} f_{\pi}} \int x^{2}(1-\cos F) d x \\
& \text { where } \\
& \mu=\frac{1}{e f_{\pi}}\left(m_{\pi}^{2}+m_{\eta}^{2}\right) \\
& \text { and } D_{88}=\frac{1}{2} \operatorname{Tr}\left(\lambda_{8} A^{\dagger} \lambda_{8} \mathrm{~A}\right) \\
& \text { Halmiltonian corresponding to Eqn. (13) is } \\
& \mathrm{H}=\mathrm{M}+\frac{1}{2}\left(\frac{1}{\mathrm{I}_{1}}-\frac{1}{\mathrm{I}_{2}}\right) \mathrm{J}(\mathrm{J}+1)+\frac{1}{2 \mathrm{I}_{2}}\left\{C_{2}[\mathrm{SU}(3)]-\frac{3}{4}\right\}-\frac{1}{2} \Upsilon_{m} D_{88}
\end{aligned}
$$

The chiral angle $\mathrm{F}(\mathrm{r})$ is taken in terms of arc-tan ansatz ${ }^{28}$ 
$\mathrm{F}(\mathrm{r})=2 \arctan \left[\left(\frac{r_{0}}{r}\right)^{2}\right]$

where $\mathrm{r}_{0}$ (the soliton size) is the variational parameter and second power of $\frac{r_{0}}{r}$ is determined by long distance behavior of eqn. of motion. After rescaling $x=e f_{\pi} r, \frac{r_{0}}{r}=\frac{x_{0}}{x}$, where $\mathrm{x}_{0}$ is the dimensionless size of soliton and is determined by minimizing the classical mass M(Eq. 14). The Eq. (14-17) can, now, be easily evaluated in terms of $\mathrm{x}_{0}$ by using Eq. (22) and gamma functions ${ }^{29-30}$.We find

$$
\begin{aligned}
& \mathrm{M}=k \frac{f_{\pi}}{e}\left[x_{0}+\frac{15}{16} \frac{1}{x_{0}}-\beta \frac{21}{16} \frac{1}{x_{0}}-\gamma \frac{15}{4} \frac{1}{x_{0}}+\frac{\mu^{2}}{3} x_{0}^{3}\right] \\
& \mathrm{I}_{1}=\frac{2}{9} \frac{k}{e^{3} f_{\pi}}\left[3 x_{0}^{2}+\frac{25}{8}-\frac{5}{8} \beta-\frac{15}{2} \gamma\right] x_{0} \\
& \mathrm{I}_{2}=\frac{2}{9} \frac{k}{e^{3} f_{\pi}}\left[4 x_{0}^{2}+\frac{9}{4}\{1-(\beta+8 \gamma)\}\right] x_{0} \\
& \Upsilon_{m}=\frac{8}{9} \frac{k}{e^{3} f_{\pi}}\left(m_{k}^{2}-m_{\pi}^{2}\right) x_{0}^{3} \\
& \text { where } k=3 \sqrt{2} \pi^{2}
\end{aligned}
$$

Minimising M (Eq.23)

$$
\mu^{2} x_{0}^{4}=\left(\frac{15}{16}-\frac{21}{16} \beta-\frac{15}{4} \Upsilon\right)-x_{0}^{2}
$$

From this eqn. one can find the soliton size $\mathrm{x}_{0}$ in terms of $\beta, \Upsilon$ and $\mu$. Using this $\mathrm{x}_{0}$, the values of $\mathrm{M}, I_{1}, \mathrm{I}_{2}$ and $\Upsilon_{m}$ can be obtained from Eqs.(23-26).From these values, baryon masses are determined ${ }^{19}$.We consider the two cases:

Case 1 . Chirally symmetric limit $\left(\mathrm{m}_{\pi}=\mathrm{m}_{\mathrm{k}}=0, \mu=0\right)$

Here, the average octet and decuplet masses are fitted ${ }^{19}$ for predicting $f_{\pi}$ and e in the following three sub-cases as:

a) $\beta=\Upsilon=0$ (Skyrme model)

$$
\begin{aligned}
& \text { one gets } \quad \mathrm{x}_{0}=\frac{\sqrt{15}}{4} \\
& \text { with } f_{\pi}=52.46 \mathrm{MeV}, \mathrm{e}=5.4 \\
& \text { b) } \beta=0, \Upsilon=0.16^{31}
\end{aligned}
$$

with these parameters, $f_{\pi}$ comes out to be negative and hence is

is not considered. It happens due to small value of strange moment of inertia $I_{2}$. For $\Upsilon=0.09$, we find

$$
\begin{array}{rl}
x_{0} & =\sqrt{3 / 5} \\
f_{\pi}=47.28 \mathrm{MeV} & \mathrm{e}=4.64
\end{array}
$$

c) $\beta=0.44^{32}, \gamma=0$

These parameters yield

$$
x_{0}=\sqrt{3 / 4}
$$

and $\quad f_{\pi}=51.7 \mathrm{MeV}, e=4.03$

Case 2. Broken chiral symmetry $(\beta=\Upsilon=0)$

$$
m_{\pi}=139 \mathrm{MeV}, m_{k=} 495 \mathrm{MeV}
$$

and $\mu^{2}=35$, we get

$$
\mathrm{x}_{0}=0.15
$$

the masses $m_{\Lambda}$ and $m_{\Sigma^{*}}$ are fitted ${ }^{19}$ which gives

$$
f_{\pi}=20.57 \mathrm{MeV}, \mathrm{e}=4.46
$$

The values obtained for $\mathrm{x}_{0}, f_{\pi}$ and $e$ in cases $1 \& 2$ will be used in calculating the hyperon decay amplitudes in section 4 .

\section{Noether Currents}

The left Noether currents in the model for lagrangian (Eq.6) can be easily obtained ${ }^{2}$. These currents for different pieces of the lagrangian are

$$
\begin{gathered}
J_{\mu, a}^{L, s k}=i \frac{f_{\pi}^{2}}{2} \operatorname{Tr}\left(\lambda_{a} \partial_{\mu} U U^{\dagger}\right)+\frac{i}{8 e^{2}} \operatorname{Tr}\left[\partial^{v} U U^{\dagger}, \lambda_{a}\right]\left[\partial_{\mu} U U^{\dagger}, \partial_{v} U U^{\dagger}\right] \\
J_{\mu, a}^{L, \beta}=-\frac{i}{8 e^{2}} \operatorname{Tr}\left[\lambda_{a}, \partial^{v} U U^{\dagger}\right]_{+}\left[\partial_{\mu} U U^{\dagger}, \partial_{\nu} U U^{\dagger}\right]_{+} \\
J_{\mu, a}^{L, \gamma}=i \frac{\gamma}{2 e^{2}} \operatorname{Tr}\left(\partial^{v} U \partial_{v} U^{\dagger}\right)\left(\operatorname{Tr} \lambda_{a} \partial_{\mu} U U^{\dagger}\right) \\
\left.J_{\mu, a}^{L, W z}=-\frac{N_{c}}{48 \pi^{2}} \varepsilon_{\mu \nu \alpha \beta} \operatorname{Tr} \lambda_{a}\left(\partial_{\nu} U U^{\dagger}\right)\left(\partial_{\alpha} U U^{\dagger}\right) \partial_{\beta} U U^{\dagger}\right]
\end{gathered}
$$

Like in ref. ${ }^{17}$ only static contributions of currents are taken in account. Terms proportional to $\dot{a}$ and $\dot{a}^{2}$ are neglected.

\section{Weak Interaction Hamiltonian}

The current-current weak interaction Hamiltonian is given by ${ }^{16-17}$

$$
H_{W}^{e f f}(\Delta S=1)=\frac{G_{F}}{\sqrt{2}} \sin \theta_{c} \cos \theta_{c} J_{L \rho}^{(1-i 2)} J_{\rho}^{\rho(4+i 5)}+\text { h.c }
$$

Consider the operator

$\hat{\mathrm{O}}(\Delta S=1)=J_{L j}^{1-i 2} J_{L j}^{4+i 5}$

By using the ansatz(Eq.10), Noether currents (sec 3), arc tan ansatz(Eq.22) and by evaluating the space integral with the help of gamma functions, we get ${ }^{16,33}$

$\hat{\mathrm{O}}(\Delta S=1)=-\varphi R_{\pi^{-} a} R_{K^{+} a}$

where

$$
\begin{aligned}
& \varphi=k \frac{f_{\pi}^{3}}{e}\left\{2 x_{0}+\frac{15}{2 x_{0}}[1-(\beta+4 \gamma)]+\frac{1}{x_{0}^{3}}\left[\frac{847}{64}\left(1+\beta^{2}\right)\right.\right. \\
& \left.\left.+\frac{693}{8} \gamma(2 \gamma-1)-\beta \frac{1078}{64}\right]\right\} \\
& R_{\pi^{-} a} R_{K^{+} a}=-\frac{3 \sqrt{6}}{5} D_{\frac{1}{2^{\prime}}, 0}^{8}-\frac{1}{10} D_{\frac{1}{2^{\prime}}, 0}^{27}-\frac{1}{\sqrt{20}} D_{\frac{3}{2}, 0}^{27}
\end{aligned}
$$

here the left lower index of SU (3) D function I $=\frac{1}{2}, \frac{3}{2}$ stands for $\left(\mathrm{YII}_{3}=\left(1 \mathrm{I}-\frac{1}{2}\right)\right.$ and right lower index for $(000)$.

The hyperon decay amplitudes can be obtained by evaluating the matrix elements of Ô$(\Delta S=1)(E q .43)$ between different hadronic states or wave functions ${ }^{34-35}$ in the model i.e.

$$
<B_{f}|\mathrm{O}(\Delta S=1)| B_{i}>
$$

with the help of SU (3) C.G. coefficients ${ }^{36}$. These matrix elements are also given in ref..$^{37}$ (table II, matrix elements of operator $\mathrm{T}_{0}$ ). Both symmetric and antisymmetric octet contribute to the matrix elements. In the transitions $B_{i} \rightarrow B_{f}$ octet dominance is exhibited because C.G. coefficients for $8 \times 8 \rightarrow 27$ and $8 \times 27 \rightarrow 8$ are smaller in magnitude than those for $8 \times 8 \rightarrow 8 \mathrm{e.g}$

$$
<P|\hat{O}(\Delta S=1)| \Sigma^{+}>=\left[(20.00)_{8}+(0.37)_{27}\right] \varphi \times 10^{-3}
$$

Our values of transition magnitudes relative to $\varphi \times 10^{-3}$ agree with those of ref. ${ }^{38}$ (table II a) and are given, here, in table 1 for easy reference. The relative values of matrix elements are given in table 2. There is a sign difference, here, for transitions $\Sigma^{0} \rightarrow N$ and $\Xi^{0} \rightarrow \Lambda^{0}$ and $\Lambda^{0} \rightarrow N$ w.r.t those in ref ${ }^{17}$. To check it, we calculate the amplitude $\mathrm{A}\left(\Sigma_{+}^{+}\right)$which in QM is zero. This amplitude is given by ${ }^{16}$ 


$$
\begin{aligned}
& A\left(\Sigma_{+}^{+}\right)=-\frac{1}{\sqrt{2}} f_{\pi}^{-1}\left[<P\left|H_{W}^{e f f}(\Delta S=1)\right| \Sigma^{+}>\right. \\
& \left.+\sqrt{2}<N\left|H_{w}^{e f f}(\Delta S=1)\right| \Sigma^{0}>\right]
\end{aligned}
$$

which yields zero by using the respective matrix elements from table 1 verifying the $\mathrm{QM}$ result.

Now, we embark on hyperon decay amplitudes. For it, first the amplitude of transition $\sum^{+} \rightarrow P$ is evaluated from Eq.(46).Then, other amplitudes can be found by using table 2 . Following two cases are taken

Case 1. Chiral symmetric limit i.e.

$$
m_{\pi}=m_{k}=0
$$

Depending on $\beta$ and $\Upsilon$, there are three sub-cases

a) $\beta=\Upsilon=0$ (Skyrme model)

By using the soliton size $x_{0}(E q .29), f_{\pi} \& e(E q .30)$ in

Eq. (44), $\varphi$ is calculated which when used in Eq.(46) gives for octet dominance, the amplitude

$A\left(\Sigma_{0}^{+}\right)=0.55 \times 10^{-3} \mathrm{GeV}^{3}$

which is about same as the Skyrme value(Eq.1) obtained numerically.

b) $\Upsilon \neq 0^{31}, \beta=0$

For $\Upsilon=0.09$, by following on lines of sub-case a, we get $A\left(\Sigma_{0}^{+}\right)=0.04 \times 10^{-3} \mathrm{GeV}^{3}$

which is less than the corresponding Skyrme value(eqn.1).

It means $\Upsilon$ term taken in the lagrangian does not improve the decay amplitudes.

$$
\begin{aligned}
c) & \beta \neq 0^{32}, \gamma=0 \\
\text { Here } \beta & =0.44 \text {, we obtain on the same lines as above } \\
& A\left(\Sigma_{0}^{+}\right)=1.35 \times 10^{-3} \mathrm{GeV}^{3}
\end{aligned}
$$

which is twice the Skyrme value(Eq.1). Therefore, $\beta$ term taken in the lagrangian produces a major change in decay amplitudes in positive direction. It was the main aim of paper to check. But the value obtained here is still less than the $\mathrm{QM}$ value(Eq.3).

Case 2. Broken chiral symmetry

$m_{\pi}=139 \mathrm{MeV}, m_{k}=495 \mathrm{MeV}$

With the parameters of Eq. (35\&36), one obtains

$$
A\left(\Sigma_{0}^{+}\right)=0.4 \times 10^{-3} \mathrm{GeV}^{3}
$$

This value shows some improvement over the Skyrme value(Eq.2)

To further check the effectiveness of $\beta$ term in the lagrangian, we calculate also the S-wave non-leptonic hyperon decay amplitudes by following the approach of ref. ${ }^{39}$ where $f_{\pi}$ is kept at its experimental value and $e=4.1$ (a suitable set of parameters for the description of baryon properties). The effective phenomenological constants take into account all QCD enhancement factors embodied into the constants $C_{i}$. With $C_{1}=2.75^{40}$ and effective hamiltonian (Eq.41),

The S-wave decay amplitudes for transition $\Sigma^{+} \rightarrow P+\pi^{0}$ corresponding to $\beta$ term in the lagrangian is

$A\left(\Sigma_{0}^{+}\right)=-3.11 \times 10^{-7}$

where $f_{\pi}=93 \mathrm{MeV}$ and $e=4.03$ which is close to 4.1 .

Our values for $\mathrm{A}\left(\Sigma_{0}^{+}\right)$is greater in magnitude than corresponding value for parameters Set $\mathrm{A}^{37}$ and is nearly equal to $\mathrm{QM}^{41}$ value. The other results for the amplitudes are summarized in table 3, where the corresponding QM values are also given. The P-wave decay amplitudes has contributions from the contact and pole terms. But the simultaneous description of both S- and P- wave decay amplitudes is not possible in the models based on chiral symmetry ${ }^{42}$. The $S$ wave/ $P$ wave amplitudes in the model will be addressed separately for the lagrangian $\beta \neq 0$ and $\gamma=0$.

\section{CONCLUSION}

We have studied $\Delta S=1$ weak transition amplitudes in the Skyrme model generalized up to quartic terms in field gradients. The analysis shows that a) the term $\gamma \neq$ $0, \beta=0$ does not show any improvement in decay amplitudes b) $\beta \neq 0$ and $\gamma=0$ term

shows major improvement in decay amplitudes over Skyrme values, still less than QM values. S-wave nonleptonic hyperon decay amplitudes calculated for $\beta$

\begin{tabular}{|c|c|c|c|}
\hline $\begin{array}{l}<B_{f}|\hat{O}| B_{i}> \\
/\left(\varphi \times 10^{-3}\right)\end{array}$ & $8\left(8_{d}+8_{f}\right)$ & $27\left(\Delta \mathrm{I}=\frac{1}{2}\right)$ & $27\left(\Delta \mathrm{I}=\frac{3}{2}\right.$ \\
\hline$P \quad \Sigma^{+}$ & 20.00 & 0.06 & 0.31 \\
\hline$N \quad \Sigma^{0}$ & $-20.00 / \sqrt{2}$ & $-0.06 / \sqrt{2}$ & $0.31 / \sqrt{2}$ \\
\hline$\Lambda \quad \Xi^{0}$ & -18.38 & 0.23 & 0.00 \\
\hline$N \quad \Lambda$ & 12.25 & 0.23 & 0.00 \\
\hline$\Sigma^{-} \Xi^{-}$ & -5.00 & 0.06 & 0.31 \\
\hline$\Sigma^{0} \quad \Xi^{0}$ & $5.00 / \sqrt{2}$ & -0.06 & $0.31 / \sqrt{2}$ \\
\hline
\end{tabular}
term agree quiet well with QM values but less than experimental values.

Table 1. Relative values of baryon matrix elements

\begin{tabular}{|c|c|c|}
\hline$<B_{f} \mid \hat{O}\left(\Delta S=1 \mid B_{i}>\right.$ & $\begin{array}{l}\text { Skyrme Model } \\
8 \text { Contribution }\end{array}$ & $\begin{array}{l}\text { Quark Model } \\
8 \text { Contribution }\end{array}$ \\
\hline$P \quad \Sigma^{+}$ & 1.00 & 1.00 \\
\hline$N \quad \Sigma^{0}$ & -0.71 & -0.71 \\
\hline$\Lambda^{0} \Xi^{0}$ & -0.91 & -0.81 \\
\hline$N \quad \Lambda^{0}$ & 0.61 & 0.41 \\
\hline$\Sigma^{-} \Xi^{-}$ & -0.25 & 0.00 \\
\hline$\Sigma^{0} \Xi^{0}$ & 0.18 & 0.00 \\
\hline
\end{tabular}
$<B_{f} \mid \hat{O}\left(\Delta S=1 \mid B_{i}>\right.$

The relative phases are so chosen that $-\Sigma^{+}$and $-\Xi^{-}$are used in $3 \times 3$ octet matrix elements

Table 2. Relative value of $<B_{f} \mid \hat{O}\left(\Delta S=1 \mid B_{i}>\right.$ compared with quark model result.

Table 3. S-wave non-leptonic hyperon decay amplitudes compared with QM and experimental values. All values are multiplied by $10^{-7}$.

\begin{tabular}{|c|c|c|c|}
\hline $\begin{array}{c}\text { Transition } \\
B_{i} \rightarrow B_{f}\end{array}$ & Our values & $\begin{array}{c}\text { Quark Model } \\
{[\mathrm{QM}] \text { values }}\end{array}$ & $\begin{array}{c}\text { Experimental } \\
\text { Values }\end{array}$ \\
\hline$\Sigma^{+} \rightarrow P\left(\Sigma_{0}^{+}\right)$ & -3.11 & -3.8 & -3.28 \\
$\Lambda^{0} \rightarrow N\left(\Lambda_{0}^{0}\right)$ & -1.90 & -1.5 & -2.37 \\
$\Sigma^{+} \rightarrow N\left(\Sigma_{+}^{+}\right)$ & 0 & 0 & 0.13 \\
$\Xi^{0} \rightarrow \Lambda^{0}\left(\Xi_{0}^{0}\right)$ & 2.86 & 3.0 & 3.43 \\
\hline
\end{tabular}




\section{REFERENCES}

[1]. T.H.R. Skyrme, Proc. Roy. Soc. A260(1961)127

[2]. G.S.Adkins, C.R. Nappi and E.Witten,Nucl.Phys.B228(1983)552

[3]. M. Mashaal,T.N. Pham and T.N.Traung, Phys. Rev. Lett. 56(1986)436

[4]. M. Mashaal, T.N. Pham and T.N.Traung, Phys. Rev. D 34(1986)3484

[5]. M. Lacombe, B. Loiseau and R. Vinh Mau Phys.Lett. 169B (1986)121

[6]. G.S.Adkins and C.R. Nappi, Phys.Lett.137B(1984)251

[7]. O.Kaymakcalan, S.Rajeev J.Schechter,Phys.Rev.D30 (1984)594

[8]. O.Kaymakcalan, J.Schechter,Phys.Rev.D31(1985)1100

[9]. G.S.Adkins,Phys.Rev.D33(1986)193

[10]. U.G.Meissner I.Zahed,Phys.Rev.Lett.56(1986)1035

[11]. A.Jackson, A.D.Jackson, A.S.Goldhaber, G.E.Brown and

L.C.Castillejo,Phys.Lett.154B(1985)101

[12]. L. Marleau, Phys. Rev.D41(1990)1606

[13]. L. Marleau, Phys. Rev.D43(1991)885

[14]. L. Marleau, Phys. Rev.D45(1992)1776

[15]. H. Weigel, Int. J. Mod.Phys.A11(1996)2419

[16]. N.N. Scoccola, Phys. Lett.428B(1998)8

[17]. M. Praszalowicz and J.Trampetic,Phys.Lett.161B(1985)169

[18]. D.Tradic and J.Trampetic,Phys.Rev.D22(1981)144

[19]. M. Praszalowicz, Phys. Lett.158B(1985)264

[20]. M.Chemtob, Nucl.Phys.B 256(1985)600

[21]. Y.Kondo, S.Saito and T.Otofuji, Phys.Lett.236B(1990)1

[22]. S. Weinberg, Physica, 96(1979)325

[23]. M. Lacombe, B. Loiseau, R.Vinh Mau and W.N.Cottingham, Phys.Lett.161B(1985)31

[24]. E. Witten, Nucl.Phys. B233(1983)422

[25]. E. Witten, Nucl.Phys. B233(1983)433
[26]. H. Weigel, Chiral Soliton Model for Baryons, Lect. Notes Phys.743(Springer, Berlin Heidelberg 2008)p.87,94

[27]. Irwandi and Triyanta, Indonesian J.Phys.17(2006)1

[28]. D.I. Diakonov, V. Petrov and M.Praszalowicz, Nucl. Phys.B 323(1989)5

[29]. G.Duplacic,H.Pasagic and J.Trampetic,JHEP 07(2004)027

[30]. Rajesh Kumar,Int.J.Mod.Phys.E 23(2014)14520028

[31]. J.F. Donogue, E.Golowich and B.R.Holestein,Phys.Rev.Lett. 53(1984)747

[32]. Rajesh Kumar and P.K. Chattopadhyay, Pramana 49(1997) 421

[33]. G. Duplancic, H.Pasagic, M. Praszalowicz and J. Trampetic, Phys. Rev.D 64(2001)097502, arXiv:hep-ph/0104281v2

[34]. E. Gaudagnini ,Nucl.Phys.B236(1984)35

[35]. A.V. Manohar, Nucl. Phys.B248(1984)54

[36]. P. McName, S.J and F.Chilton,Rev.Mod.Phys.36(1964)1005

[37]. N. Toyota, Prog. Theo.Phys.77(1987)688

[38]. N. Toyota and K.Fuji,Prog.Theo.Phys.75(1986)340

[39]. Daniel Gomaz Dumm, A.J.Gracia and N.N.Scoccola Phys. Rev.D62(2000)014001

[40]. M.A.Shifman, A.J.Vainstein and V.I. Zakharov, Nucl. Phys.B 120(1977)317

[41]. J.F. Donoghue, E. Golowich and B.R.Holstein,Phys.Rep.131 (1986)319

[42]. E. Jenkins, Nucl. Phys.B375(1992)561, hep$\mathrm{ph} / 9508324$

Cite this article as : Rajesh Kumar, " $\Delta S=1$ Weak Transitions in Skyrme model Generalized up to Quartic Terms", International Journal of Scientific Research in Science and Technology (IJSRST), Online ISSN : 2395602X, Print ISSN : 2395-6011, Volume 7 Issue 2, pp. 558562, March-April 2020. Available at Journal URL : http://ijsrst.com/IJSRST207290 